Edited by Kiriakos Xenitidis and
Colin Campbell
Contents
- Skype and narcissistic disturbances: a unique
opportunity?
- Anxiety and mortality in the elderly
- Does assessing quality of life improve patient
satisfaction? Yes - unless you look at the results

\section{Skype and narcissistic disturbances: a unique opportunity?}

Psychiatrists are increasingly incorporating advances in communications into their clinical practices. Skype is an example of one treatment medium which has been adopted by many practitioners and has been recognised for its capacity to provide enhanced convenience, to increase access for patients and to allow for continuity of care. ${ }^{1}$ Despite lingering questions regarding the Health Insurance Portability and Accountability Act 1996 (HIPPA) compliance and licensure requirements, reports indicate that a substantial number of mental health professionals are currently using non-traditional communication media in their practices. ${ }^{2}$

The increasing utilisation of voice-over-internet methods such as Skype by mental health providers has resulted in the emergence of novel therapeutic matrices with attendant challenges and opportunities. In this letter, I describe how some of the features of Skype, and video conferencing more generally, offer a unique opportunity to address therapeutically a fundamental component of the narcissistic disturbances.

Disturbances in the experience of the self are central to narcissistic vulnerabilities. Kohout believed that parental empathic failures resulted in a child's inability to successfully modulate fundamental self-functions including self-esteem and mood and to be dependent on others (self-objects) to mediate these functions. ${ }^{3}$ Kohout thought that psychotherapeutic treatment of such narcissistic disturbances required therapeutically focusing attention on empathy and in particular on analysing and working through empathic failures that occurred between the therapist and patient over the course of treatment.

Skype and similar video conferencing technologies present opportunities to explore empathy, connection, attunement and their vicissitudes. In traditional therapy, empathic failures that inevitably arose over the course of treatment - the therapist being late or not responding to a comment in an empathic way - tended to be addressed as they materialised. However, the intrinsic features of video conferencing such as connection strength, the complexities involved with consistently achieving direct eye contact, not infrequent problems with audio and visual components resulting in less than optimal images, missed words and delays together create empathic failures and misattunements in the relational field and thrust such issues, particularly disconnects, to the forefront of treatment.

In my practice I have found that such actualities of Skype serve as valuable opportunities to explore these technologically facilitated empathic mismatches. Early in treatment, such Skypeassociated empathic divergences need to be addressed at a manifest level such that a complaint about sound quality should result in a collaborative attempt to remedy the audio issue. Demonstrating to the patient one's concern about the connection and willingness to help remedy it demonstrates responsiveness. Later in treatment, patients' feelings about the disconnect can be more fully explored, for instance 'How did you feel when you could not hear me?' In this way, a progressive approach involving early responsiveness to, and later further discussion of, technologically facilitated empathic mismatches can help necessary work on patients' underlying narcissistic issues.

1 Hoffman J. When your therapist is only one click away. The New York Times 2011; 23 September.

2 Jacobsen T, Kohout J. 2008 APA Survey of Psychology Health Service Providers: Telepsychology, Medication and Collaboration. American Psychological Association, 2010 (http://www.apa.org/workforce/ publications/08-hsp/telepsychology/report.pdf).

3 Baker HS, Baker MN. Heinz Kohut's self psychology: an overview. Am J Psychiatry 1987; 144: 1-9.

Geoffrey B. Neimark, Department of Psychiatry, University of Pennsylvania, USA. Email: gneimark@hotmail.com

doi: $10.1192 /$ bjp.204.1.79

\section{Anxiety and mortality in the elderly}

Carrière et al's study shows an interesting association between anxiety and mortality in elderly women. ${ }^{1}$ The authors propose a series of possible biological mechanisms for this association, suggesting a direction of causality in which mortality is the consequence of the impact that anxiety has on the endocrine and cardiovascular systems. However, anxiety can be the psychiatric expression of vascular changes in the brain that may eventually lead to death. Failing health in old age is also a painful reminder of the proximity of death, which will frequently induce feelings of anxiety in the individual. The fact that this association was only significant for women could be an artefact due to the much higher prevalence of anxiety among women. Thus, anxiety may well be - at least in a proportion of the cases - the consequence, rather than the cause of ill health.

1 Carrière I, Ryan J, Norton J, Scali J, Stewart R, Ritchie K, et al. Anxiety and mortality risk in community-dwelling elderly people. Br J Psychiatry 2013; 203: 303-9.

Rafael Euba, Oxleas NHS Foundation Trust, London, UK.

Email: Rafael.Euba@oxleas.nhs.uk

doi: 10.1192/bjp.204.1.79a

Authors' reply: We agree that in observational studies residual confounding bias may subsist. However, to take into account this potential drawback with anxiety being a consequence of prior vascular changes, we carefully adjusted the models for a large number of confounding factors including vascular risk factors and cardiovascular diseases, and the association in women remained significant. The second argument of failing health and proximity of death does not hold as at baseline (time of anxiety evaluation) our sample consisted of high-functioning community-dwelling elderly persons, physically and psychologically able to travel to the medical centre. A careful examination of the Kaplan-Meier curves (Fig. 1) also indicates that very few deaths occurred during the first 2 years of follow-up. Last, Euba raises the question of statistical power to explain the absence of a significant association in men. In survival analysis, the statistical power depends on the number of events (i.e. deaths) and in our 
sample the frequency of deaths is higher in men (162/702 v. 136/ 1006 in women). A power calculation for anxiety disorder shows that we could have detected an unadjusted relative risk of 1.65 in women and $1.71 \mathrm{in}$ men, with an alpha risk of 0.05 and a power of 0.80 . The analysis in men is thus not underpowered and if an association with mortality exists in men, it is less strong than in women.

With ageing, people face multiple adverse events including physical multimorbidity and loss of capacities. Personal resources, such as self-efficacy, sense of mastery or control beliefs, and psychological resilience are important in the process of coping with a chronic disease. On the other hand, anxiety disorder, irrespective of the aetiology, could clearly contribute to a worse outcome. This underlines the importance of developing interventions for older persons aimed at maintaining or improving psychological coping resources when health declines. Up to now, very few well-designed studies have been performed with such a large population sample, capable of controlling for main confounders and using a validated anxiety diagnosis including anxiety subtypes. Although future research is needed to confirm our results and the gender-specific association, our study also stresses the importance of including anxiety diagnostic tools in population-based cohorts to improve the understanding of the consequences of anxiety in late life.



doi: 10.1192/bjp.204.1.79b

\section{Does assessing quality of life improve patient satisfaction? Yes - unless you look at the results}

The need for holistic assessment in psychiatry is becoming ever more pertinent. Therefore, we were pleased to read Boyer et al's randomised controlled trial. ${ }^{1}$ The team investigated whether assessing and feeding back a quality of life (QoL) measure to the patient's care team as part of a psychiatric assessment would improve patient satisfaction when compared with both standard psychiatric assessment and with measuring QoL but not informing the care team of the results.

We were also initially pleased to read that 'global satisfaction was significantly higher in the QoL feedback group [...] compared with the standard psychiatric assessment [...] and QoL assessment groups. However, on closer inspection, it appears that this interpretation of the results is not correct.

The primary outcome was level of patient satisfaction in the QoL feedback group compared with standard psychiatric assessment. In this comparison, 29 out of 40 patients $(72.5 \%)$ in the QoL feedback group were 'very satisfied' with their care, compared with 27 out of $40(67.5 \%)$ in the control group. This difference of $5 \%$ is far too small to be statistically significant with the sample size used.

Indeed, when we undertook our own basic statistical analysis, we found that the $95 \%$ CIs for relative risk ratio between these two groups were $-37 \%$ to $22 \%$ - clearly not significant.

The correct interpretation of these results is that the study actually provides no evidence for assessing and feeding back a QoL measure in preference to simply not measuring QoL at all. The conclusions drawn by the authors, that their findings 'provide strong support for integrating QoL assessment and feedback' and that 'priority should be given to strategies to implement QoL measurements in routine practice' seem particularly unfounded.
Although we agree that QoL measures represent a potentially highly useful clinical tool, we cannot accept that Boyer et al's study provides evidence for this claim in any way.

We felt that the most salient finding from the trial was in fact the far lower satisfaction in the control group of patients who had their QoL assessed but had the results ignored. If we offer an assessment or intervention, we should be careful to follow-up our intentions or the result may actually be detrimental overall.

1 Boyer L, Lançon C, Baumstarck K, Parola N, Berbis J, Auquier P. Evaluating the impact of a quality of life assessment with feedback to clinicians in patients with schizophrenia: randomised controlled trial. Br J Psychiatry 2013; 202: 447-53.

Alexander E. Langford, Douglas Badenoch, South London and Maudsley NHS Foundation Trust, London, UK. Email: alexander.langford@slam.nhs.uk

doi: $10.1192 /$ bjp.204.1.80

Authors' reply: We are in agreement with Langford \& Badenoch's general comment on the need for holistic assessment in psychiatry. It is currently established that patients' views, and especially quality of life (QoL) measures, should supplement the usual indicators of quality in healthcare. ${ }^{1}$ However, we are doubtful about the relevance of their criticisms.

Langford \& Badenoch denounced the following sentence: 'Global satisfaction was significantly higher in the QoL feedback group [. . .] compared with the standard psychiatric assessment [...] and QoL assessment groups.' This assumption was derived, however, directly from our results (i.e. the proportions of very satisfied patients were $73 \%$ in the QoL feedback group, $45 \%$ in the QoL assessment group and 68\% in the standard group). The comparison performed using a chi-squared test was statistically significant $(P=0.025)$, allowing us to state that global satisfaction significantly differed between the three groups. As we have written in our Discussion, this finding did not prohibit us from suggesting that integrating QoL assessment and feedback with standard psychiatric assessment seemed relevant or that priority should be given to strategies that implement QoL measurements in routine practice.

Moreover, this assumption was in agreement with our study design (i.e. three arms) and the sample size calculation performed for this design. However, we recognise that multiple treatment arms in randomised controlled trials (RCTs) are sources of misunderstanding, ${ }^{3}$ especially because there are several possible comparisons. ${ }^{4}$ Langford \& Badenoch re-wrote our primary outcome for a two-arm RCT as follows: 'level of patient satisfaction in the QoL feedback group compared with standard psychiatric assessment', implying pairwise chi-squared tests. However, our primary outcome and analysis were defined in accordance with the primary objective integrating the three-arm design. The objective was to globally determine the 'positions' of QoL feedback, QoL without feedback and the control group with respect to their relationships to satisfaction; we did not aim to question the relevance of using the QoL measure (which is already recognised in the literature) in $2 \times 2$ comparisons between the different arms. The primary criterion was thus analysed using a global chi-squared test, determined a priori; it was not analysed using pairwise chi-squared tests (as recommended by Langford \& Badenoch), which were not planned and for which the alpha error risk was not controlled. It is also widely recognised that bias may be introduced if decisions regarding data analysis are driven by the data. ${ }^{3}$

Langford \& Badenoch also claim that 'The conclusions drawn by the authors, that their findings "provide strong support $[\ldots]$ " 